\title{
Spectroscopic properties of a two-level atom interacting with a complex spherical nanoshell
}

\author{
Alexander Moroz* \\ Wave-scattering.com
}

November 5, 2018

\begin{abstract}
Frequency shifts, radiative decay rates, the Ohmic loss contribution to the nonradiative decay rates, fluorescence yield, and photobleaching of a two-level atom radiating anywhere inside or outside a complex spherical nanoshell, i.e. a stratified sphere consisting of alternating silica and gold concentric spherical shells, are studied. The changes in the spectroscopic properties of an atom interacting with complex nanoshells are significantly enhanced, often more than two orders of magnitude, compared to the same atom interacting with a homogeneous dielectric sphere. The detected fluorescence intensity can be enhanced by 5 or more orders of magnitude. The changes strongly depend on the nanoshell parameters and the atom position. When an atom approaches a metal shell, decay rates are strongly enhanced yet fluorescence yield exhibits a well-known quenching. Rather contra-intuitively, the Ohmic loss contribution to the nonradiative decay rates for an atomic dipole within the silica core of larger nanoshells may be decreasing when the silica core - inner gold shell interface is approached. The quasistatic result that the radial frequency shift in a close proximity of a spherical shell interface is approximately twice as large as the tangential frequency shift appears to apply also for complex nanoshells. Significantly modified spectroscopic properties (see computer program freely available at http://www.wave-scattering.com) can be observed in a broad band comprising all (nonresonant) optical and near-infrared wavelengths.
\end{abstract}

PACS numbers: 78.67.Bf, 33.70.Jg, 32.70.Jz, 33.50.-j, 87.64.Ni, 87.64.Xx

*http://www.wave-scattering.com 


\section{Introduction}

Spectroscopic properties of an isolated atom, such as radiative and nonradiative decay rates, frequency shifts, and fluorescence yields are not inherent to the atom but characteristic of an atom coupled to a physical system. Indeed, it has been known for a long time that the presence of a small structure, cavity, or an interface can significantly impact the characteristic behaviour of a radiating system, irrespective if the emission takes place inside or adjacent to a material body [1, 2, 3. The physical origin of the decay of an excited atom state is the coupling of the atom to the vacuum electromagnetic field. A nearby presence of a material body modifies the vacuum electromagnetic field at the atom position. Consequently, the atom interacts with the modified vacuum electromagnetic field and it will exhibit different spectroscopic properties than the same atom in the absence of the material body. There is growing interest in the application of various systems that can significantly affect the vacuum electromagnetic modes. Such systems are currently of great interest in the fields of photonics and quantum electrodynamics. They have found widespread application in microcavity lasers, electroluminescent devices, and proposed photonic band-gap devices. There is also growing interest in the application of various aspects of the moleculesurface interaction in the field of medical diagnostics, particularly in the immuno-assay area, in which fluorescence-based techniques are widely used [4. Alternatively, for the applications in near-field optical microscopy, one is interested in changes in the atom fluorescence properties induced by the presence of a nearby microscope tip.

For the purpose of this article, the atom would mean any localized fluorescent dipole source, e.g., fluorescent organic group, rare earth atom, etc. The atom would be considered as a two-level system in the regime of weak coupling, within the domain of applicability of the linear response theory [5, 6, 7, 8]. In the latter case, the quantum-mechanical description [5, 6, 7, 8, yields identical results to the classical description [9, 10, 11, 12, 13. 14. The spectroscopic properties of the atom will be studied as a function of the atom position inside and outside stratified spheres. The theory of fluorescence properties of an atomic dipole has has been mostly investigated only in the case of a homogeneous sphere [7, 8, 10, 11, 13, 14, 15. Chew et al [16] provided a formal solution to the problem of a dipole radiating in the presence of a multilayered sphere. However, their solution for the sphere with $N$ concentric shells (the sphere core counts as shell number one) is written in terms of a $2 N \times 2 N$ matrix and appears awkward and impractical for numerical calculations. Indeed, neither Chew nor anybody else have appeared to implement the Chew et al [16] solution numerically. The main obstacles are that as $N$ increases so do computer memory requirements to store the matrix and the time to carry out the matrix calculations, which increases as $N^{3}$. Whenever a radiating dipole has been discussed interacting with a multicoated sphere, either the problem has been treated in a quasistatic approximation [3], or the dipole has only been allow to radiate at the sphere origin [7, 17, 18, 19, 20]. The dipole position in the center of such a complex sphere considerably simplifies calculation as the inherently vector problem reduces to the scalar one involving scalar fields $\mathbf{r} \cdot \mathbf{B}(\mathbf{r})$ and $\mathbf{r} \cdot \mathbf{D}(\mathbf{r})$ [17, 18]. Additionally, in most cases only the simplest 
core-shell particles have been dealt with [3, 7, 19, 20]. Although Li et al [21] has provided a recursive formula for Green's function for the case of a multicoated sphere with an arbitrary number of concentric shells, an efficient numerical use of Li's formula, even for coinciding spatial arguments, requires to perform traces over the magnetic angular momentum number. Only very recently the limitation on the dipole position at the center of a general multilayered sphere has been removed and the traces over the magnetic angular momentum number in the scattering Green's function at coinciding spatial arguments, $\mathbf{G}(\mathbf{r}, \mathbf{r}, \omega)$, have been explicitly performed [12. A complete description of the classical electromagnetic fields of a radiating electric dipole has been achieved outside and inside a multi-structured spherical particle. Electromagnetic fields have been determined anywhere in the space, and the time-averaged angular distribution of the radiated power, the timeaveraged total radiated power, radiative and nonradiative (due to Ohmic losses) decay rates, frequency shifts have been calculated. Our recursive solution only employs $2 \times 2$ transfer matrices and their ordered products and provides a fast and reliable algorithm which can easily be implemented numerically [12].

In the present article, the theory developed in Ref. [12] will be applied to "nanomatryoshka" structures of Prodan et al [22, i.e., multilayered spheres consisting of alternating silica and gold concentric spherical shells. Such complex spheres have also been known as nanoshells. Current experimental colloidal techniques allow one to design a variety of multi-structured beads having a plurality of concentric shells with the core radius from cca $1 \mathrm{~nm}$ till $1 \mu \mathrm{m}$ and controlled shell thicknesses. For instance, metal (Au, $\mathrm{Ag}, \mathrm{Pt})$ and dielectric $(\mathrm{ZnS})$ beads can be coated in a controlled way by a silica shell [23. 24, 25, 26, 27, and a dielectric (silica, $\mathrm{Au}_{2} \mathrm{~S}$ ) bead can be coated by gold or some other noble metal 22, 28, 29, 30, 31, 32. One can subsequently etched away silica core of a silica-core metal-shell bead and obtain a hollow metallic nanoshell. Either hollow metallic nanoshell or a dielectric-core metal-shell bead can be in turn coated in a controlled way by the second concentric silica shell (a dielectric overcoat of either metallic shell or a metallic core prevents aggregation of the particles by reducing the Van der Waals forces between them) [22, 31] and by a further metal shell, thereby forming a "nano-matryoshka" [22]. Compared to a simple homogeneous sphere, such a complex multilayered spherical particles allows one a lot more freedom in engineering of both elastic [22, 28, 29, 30, 33. and inelastic light-scattering properties [34]. Many other examples of stratified spheres can also be found in nature. As an example, water insoluble aerosols in atmosphere have a thin liquid layer adsorbed on their surface. With the addition of an appropriate surfactant, water droplets in different hydrophobic solvents (such as oil) form a stable nanometer sized structures (with the size depending on the water to surfactant molar ratio), often referred to as "reversed micelles", consisting of a spherical water core coated with a surfactant monolayer [3]. In the case of a biological cell, the appropriate model consists of concentric three-layered sphere, corresponding to nucleus, cytoplasm, and membrane [16]. The case of a sphere having two coatings is also important for modeling hydrological particles coated with biological material and micro-encapsulated material.

A unique feature of "nano-matryoshka" structures of Prodan et al [22], which partly explains our focus on these structures, is the existence of two coupled nanocavities sur- 
rounded by metal boundaries (see Fig. 1). The latter feature holds promise of large and controlled tunability of light-matter interactions, including both the "nano-matryoshka" scattering properties and the spectroscopic properties of the atom interacting with such a nanostructure. As it will be shown below, such a complex nanoshell geometry strongly affects spontaneous emission decay rates, $W^{\text {rad }}$, photostability, and the ratio $W^{r a d} / W^{\text {tot }}$, known as the fluorescence yield, or simply, quantum efficiency (here $W^{\text {tot }}=W^{r a d}+W^{\text {nrad }}$ denotes the total decay rate) [20]. The spontaneous emission rate $W^{\text {rad }}$ is one of three Einstein's coefficients (usually denoted as A coefficient). The remaining two Einstein's coefficients (usually denoted as B coefficients) describe the stimulated emission probability and the absorption probability. When multiplied by the radiant energy density $U_{\omega} d \omega$ with circular frequency between $\omega$ and $\omega+d \omega$, the respective B coefficients then determine the stimulated emission and absorption rates at the transition frequency $\omega$. Detailed balance in thermal equilibrium implies that the knowledge of a single Einstein coefficient is sufficient to determine the remaining two. Therefore, once the spontaneous emission rate is known, the stimulated emission and absorption rates are also unambiguously determined. For instance, the stimulated emission probability and the absorption probability are equal and the ratio of spontaneous to stimulated emission decay rate remains equal to the mean thermal photon number at the transition frequency, a constant which does not change with changing environment (see third reference in [1]). Thus, an inhibited (enhanced) spontaneous emission necessarily implies inhibited (enhanced) stimulated emission by the same factor. There is hope that using complex nanostructures one would be able to tailor spontaneous and stimulated decay rates according to one's need and a desired application, such as chemical speciation, LIDAR, fluorescent near-field microscopy, identification of biological particles, and monitoring specific cell functions. In this respect, fluorescent properties of the atom both inside and outside of a complex nanostructure are of fundamental interest. For instance, by placing fluorescent organic groups or rare earth ions (with nm control over the radial position [26]) inside the dielectric core or dielectric shell of such a complex nanoparticle 32, a fluorescent nanoprobe can be formed for biophysical and biomedical applications [4. Alternatively, for the applications in near-field optical microscopy [35, wherein the probe tip is modeled as a sphere of small radius, one is interested in changes in the atom fluorescence properties induced by the presence of a nearby complex nanoparticle.

\section{Theory}

In order to characterize the change in the spectroscopic properties of the atom interacting with a (complex) spherical scatterer, the frequency shifts, radiative and nonradiative decay rates will all be normalized with respect to the radiative decay rates of the same dipole but now in a free-space filled in with a homogeneous medium which is identical to that at the atom position. Such a normalization of spectroscopic properties brings an advantage that, in the case of radiative decay rates, any local field corrections [2, 3, 18, 36] cancel

out. In the case of a homogeneous dielectric medium (characterized by the refractive 
index $n$ and dielectric permittivity $\varepsilon$ ) Nienhuis and Alkemade [37] have showed that

$$
W_{h}^{r a d}=\frac{n^{3}}{\varepsilon} W_{v}^{r a d}
$$

where $W_{h}^{r a d}$ and $W_{v}^{\text {rad }}$ are radiative rates of the electric-dipole transitions in the dielectric medium and in the vacuum, respectively. For a non-magnetic medium, the above relation reduces to $W^{r a d}=n W_{0}^{r a d}$. It is emphasized here that these results holds irrespective if a homogeneous medium is dispersive or not. (For the general case of a linear, nonconducting, absorptive, and dispersive medium see Sec. VIII of Ref. 38.)

Formally, irrespective of either the classical or quantum-mechanical descriptions, the line broadening and frequency shift of an electric-dipole emitter interacting (via the vacuum electromagnetic field) with a material body can be understood as a result of the coupling of the emitted field with its own reflected field. Let us label the concentric shells of a complex nanoshell from the nanoshell core outward, with the nanoshell core counting as the shell number one and the ambient counting as the shell number five. Let $r_{j}$, $j=1,2,3,4$, and $\varepsilon_{j}, \mu_{j}, k_{j}=\omega_{0} \sqrt{\varepsilon_{j} \mu_{j}} / c, j=1,2,3,4,5$, denote the respective shell radii, dielectric permittivities, and wave vectors. Occasionally, as in Fig. 1 the subscript 5 will be replaced by $h$ to indicate the host medium (ambient). Then, within the linear response formalism of Agarwal [5] and of Wylie and Sipe [6], the effective shift in the frequency separation $\omega_{0}$ of two levels of the atom within the $n$-th shell is given as follows (see Refs. [5, 7, 8, 9, 13, ),

$$
\frac{\omega-\omega_{0}}{W_{h}^{r a d}}=-\frac{3 \varepsilon_{n}}{4 p^{2} k_{n}^{3}} \operatorname{Re}\left[\mathbf{p} \cdot \mathbf{G}\left(\mathbf{r}_{d}, \mathbf{r}_{d}, \omega_{0}\right) \cdot \mathbf{p}\right]=-\operatorname{Re} \frac{3 \varepsilon_{n}}{4 p^{2} k_{n}^{3}} \mathbf{p} \cdot \mathbf{E}_{s}\left(\mathbf{r}_{d}, \omega_{0}\right) .
$$

Here $\mathbf{r}_{d}$ is the dipole position, $\mathbf{p}$ is the transition dipole moment, $k_{n}=\omega_{0} \sqrt{\varepsilon_{n} \mu_{n}} / c$, and $\mathbf{G}\left(\mathbf{r}, \mathbf{r}_{d}, \omega\right)$ denotes the scattering Greens function normalized such that the electric field $\mathbf{E}_{s}(\mathbf{r}, \omega)$ of the scattered radiation at $\mathbf{r}$ due to a dipole $\mathbf{p}$ radiating at frequency $\omega$ at $\mathbf{r}_{d}$ is given by

$$
\mathbf{E}_{s}(\mathbf{r}, \omega)=\mathbf{G}\left(\mathbf{r}, \mathbf{r}_{d}, \omega\right) \cdot \mathbf{p} .
$$

For a homogeneous sphere one obtains in the quasistatic limit [13, 14,

$$
\begin{aligned}
\left(\frac{\omega-\omega_{0}}{W_{h}^{r a d}}\right)_{\|} & =\frac{3}{32} \frac{\varepsilon_{1}-\varepsilon_{2}}{\varepsilon_{1}+\varepsilon_{2}} \frac{1}{\left(k_{2} r_{s}-k_{d} r_{d}\right)^{3}}, \\
\left(\frac{\omega-\omega_{0}}{W_{h}^{r a d}}\right)_{\perp} & =\frac{3}{16} \frac{\varepsilon_{1}-\varepsilon_{2}}{\varepsilon_{1}+\varepsilon_{2}} \frac{1}{\left(k_{2} r_{s}-k_{d} r_{d}\right)^{3}},
\end{aligned}
$$

where $k_{d}$ is the radiation wave vector in the medium wherefrom the dipole is radiating. The most characteristic feature of the quasi-static approximation is that the frequency shift is a monotonic function of the dipole distance from the sphere boundary. Moreover, for the limiting cases of an atomic dipole in a close proximity to the sphere and in the long-wavelength limit the quasi-static approximation fails to account for the retardation effects and the radiative decay rate (linewidth) remains unchanged [13, 14]. 
Whereas the change in the effective shift in the frequency separation of two levels is given in terms of the real part of $\mathbf{G}\left(\mathbf{r}, \mathbf{r}, \omega_{0}\right)$, the total decay rate induced by the presence of a (multilayered) sphere is determined by the imaginary part of $\mathbf{G}\left(\mathbf{r}, \mathbf{r}, \omega_{0}\right)$. Indeed, within the linear response formalism of Agarwal [5] and of Wylie and Sipe [6], the normalized decay rate for the atom within the $n$-th shell is given as [5, 7, 8, 9, 10, 13, 18,

$$
\frac{W^{t}}{W_{h}}=1+\frac{3 \varepsilon_{n}}{2 p^{2} k_{n}^{3}} \operatorname{Im}\left[\mathbf{p} \cdot \mathbf{G}\left(\mathbf{r}_{d}, \mathbf{r}_{d}, \omega_{0}\right) \cdot \mathbf{p}\right]=1+\operatorname{Im} \frac{3 \varepsilon_{n}}{2 p^{2} k_{n}^{3}} \mathbf{p} \cdot \mathbf{E}_{s}\left(\mathbf{r}_{d}, \omega_{0}\right)
$$

The basic assumption is, of course, that neither the transition matrix element nor the transition frequency are appreciably changed by the presence of the interface.

In the presence of an absorption, as in our case, the decay rate $W^{t}$ comprises the following two basic decay channels: 1) the process of real (i.e., not virtual) photon emission with the photon escaping to the spatial infinity, i.e., radiative decay, and 2) the process of real (i.e., not virtual) photon emission accompanied by the subsequent photon absorption by the microsphere, i.e., a nonradiative decay, [5, 7, 8, 9, 10, 13, 18]. In an ideal theoretical situation (i.e., a single fluorescent atom, the respective silica and gold shell being without any impurities, and multi-photon relaxation absent) the decay rate $W^{t}$, as calculated according to Eq. (5), would be the total spontaneous decay rate. However, the Ohmic loss is only one of many other nonradiative mechanisms, such as, for instance, multiphoton relaxation, coupling to defects, direct electron-transfer processes, and concentration quenching, which may all contribute to the nonradiative decay rate $W^{\text {nrad }}$ [39, 40, 41, 42, 43] but are not included in Eq. (5). Therefore, in practice, $W^{t}$ would be the lower limit to the total spontaneous decay rate $W^{\text {tot }}$.

Obviously, in the absorbing case, the spontaneous decay rate $W^{t}$, as calculated according to Eq. (5) from the imaginary part of Green's function at coinciding arguments, $\mathbf{G}(\mathbf{r}, \mathbf{r}, \omega),\left[7,8\right.$ ] does not coincide with the radiative decay rate $W^{\mathrm{rad}}$. The latter can be, up to a proportionality factor, determined as the classically radiated power of a dipole which escapes to spatial infinity, or simply the radiative loss, $P^{\text {rad }}$, which a classical dipole experiences when interacting with a (multicoated) sphere [10, 11, 12, 15. $P^{\text {rad }}$ is calculated from the electromagnetic flux given by the surface integral of the Poynting vector through a virtual sphere of radius $R$ extending to infinity 10, 11, 12. According to the correspondence principle, the radiative decay rates $W^{\text {rad }}$ is then given by

$$
W^{r a d}=\frac{P^{r a d}}{\hbar \omega}
$$

In the absence of absorption, $W^{t}$ and $W^{r a d}$ coincide. In the presence of an absorption, the ratio $W^{r a d} / W^{t}$, also known as fluorescence yield, is always smaller than one. The relative difference between $W^{\text {rad }}$ and $W^{t}$ is especially pronounced in the proximity of metal boundaries (see Fig. 10 below). The quantum theoretical expression for the power radiated by the spontaneous emission from an excited state in an electric (a magnetic) dipole transition is still obtained from the classical expression for the power radiated by an electric (a magnetic) dipole, by replacement of the dipole moment by the corresponding 
transition matrix element. (An expression for the dipole source intensity detected by a point detector has been provided by Dung et al [8] (see Eqs. (34-36) therein).)

Let $k_{0}$ be the vacuum wave vector and $\varepsilon^{\prime \prime}\left(\mu^{\prime \prime}\right)$ be the imaginary part of the dielectric function (magnetic permeability) at the observation point. The Ohmic loss contribution to the nonradiative decay rates, $P^{n r a d}$, is calculated according to formula

$$
P^{n r a d}=\int_{a} Q(\mathbf{r}) d \mathbf{r}
$$

where the volume integral extends over all the absorbing regions. $Q$ is given as the steady (averaged) inflow of energy per unit time and unit volume from the external sources which maintain the field,

$$
Q=\frac{c k_{0}}{8 \pi}\left(\varepsilon^{\prime \prime}|\mathbf{E}|^{2}+\mu^{\prime \prime}|\mathbf{H}|^{2}\right) .
$$

Here the averaging is performed with respect to time and assuming that the amplitude of a monochromatic electromagnetic field is a constant. The formula (8) for Ohmic loss density remains also valid in the regions of high absorption near resonance frequencies of the permittivity and permeability, even when the so-called Brillouin expression for the electromagnetic field energy density,

$$
U=\frac{1}{8 \pi}\left[\mathbf{E} \cdot \mathbf{E}^{*} \frac{d[\omega \varepsilon(\omega)]}{d \omega}+\mathbf{H} \cdot \mathbf{H}^{*} \frac{d[\omega \mu(\omega)]}{d \omega}\right]
$$

is no longer valid (see Appendix $\mathrm{C}$ of Ref. [12]). For simplicity, we will assume that $\mu^{\prime \prime} \equiv 0$, i.e., the Ohmic losses will be entirely determined by an integral of the squared amplitude of the electric intensity (see Ref. [12] for calculational details).

\section{Results}

In this section, detailed results of numerical simulations are shown for "nano-matryoshka" structures of Prodan et al [22], i.e., multilayered spheres with silica core and surrounded by three additional concentric spherical shells: an inner gold shell, followed by a silica spacer layer, and terminated by an outer gold shell. As in Ref. [22], "nano-matryoshka" structures have been considered with the following dimensions: $r_{1} / r_{2} / r_{3} / r_{4}: 80 / 107 / 135 / 157$ $\mathrm{nm}$ (A), 77/102/141/145 nm (B), and 396/418/654/693 nm (C). As comparative examples, the results are also presented for a homogeneous silica sphere with radius $r_{s}=150$ $\mathrm{nm}(\mathbf{D})$, a homogeneous gold sphere with radius $r_{s}=693 \mathrm{~nm}(\mathbf{E})$, and a homogeneous gold sphere with radius $r_{s}=150 \mathrm{~nm}(\mathbf{F})$. The radius of the sphere $\mathbf{E}$ was chosen to coincide with that of the sphere $\mathbf{C}$, whereas the radius of the spheres $\mathbf{D}$ and $\mathbf{F}$ was selected to lie between that of the spheres $\mathbf{A}$ and $\mathbf{B}$. The radiating wavelength was taken to be 595 $\mathrm{nm}$, implying gold refractive index $n_{A u} \approx 0.248+i 2.986$ [44. All the spheres are assumed to be suspended in an aqueous solution. The respective refractive indices of silica and water are assumed to be $n_{\mathrm{SiO}_{2}}=1.45$ and $n_{\mathrm{H}_{2} \mathrm{O}}=1.33$. There is nothing particular in choosing the wavelength of $595 \mathrm{~nm}$, except of it being an emission wavelength of lissamine 
molecules [4]. Any other (nonresonant) optical wavelength would lead to qualitatively similar conclusions. The choice of water as an ambient has been motivated by a fact that (i) this often corresponds to an experimental situation and (ii) aqueous solution matches biological conditions.

In numerical simulations, the angular-momentum cutoff value of $l_{\max }=60$ was used. In the case of the total and radiative decay rates, the cutoff value was sufficient to obtain convergence on at least 8 significant digits (see Fig. 8 of Ref. [12]). In the case of the Ohmic loss contribution, an immediate metal shell proximity provides a numerical challenge. However further away from metal interfaces the convergence of up to at least 8 significant digits can be attained again (see Fig. 9 of Ref. [12]).

\subsection{Frequency shifts}

The radiative frequency shifts have been calculated directly from the real part of Green's function at coinciding arguments according to Eq. (2) (see also Eq. (137) of Ref. [12]). The dependence of the frequency shifts on an atomic dipole position inside and outside the "nano-matryoshka" structures A-C and the homogeneous spheres $\mathbf{D}-\mathbf{F}$ for the respective radial and tangential dipole orientations is shown in Figs. 2, 3. Since in the sphere center the difference between the radial and tangential orientation of a dipole disappears, the corresponding radial and tangential quantities coincide there.

In order to appreciate changes in the frequency shifts induced by nanoshells $\mathbf{A}-\mathbf{C}$ geometry, let us first discuss the comparative example $\mathbf{D}$ of a homogeneous silica sphere. Inside the dielectric sphere $\mathbf{D}$, the frequency shift of a radially oriented dipole steadily increases from its value of $\approx 0.0117$ at the sphere origin till that of $\approx 261$ at $r / r_{s}=$ 0.995075 (the last sampled point inside the spheres). On the other hand, the relationship between the frequency shift and the position of a tangentially oriented atomic dipole has an oscillating character, first decreasing from the value of $\approx 0.0117$ at the sphere origin down to $\approx 0.00784$ at $r / r_{s}=0.388$, and subsequently steadily increasing till $\approx 133$ at $r / r_{s}=0.995075$. (Though due to the scale of ordinate axis, the shift appears to be a flat featureless horizontal line). Outside the dielectric sphere $\mathbf{D}$, it is the frequency shift of the radially oriented dipole which exhibits an oscillating behaviour, increasing from the value of $\approx-334$ at the very first sampled point outside the sphere at $r / r_{s}=1.005025$, reaching the maximum of $\approx 0.0038$ at $r / r_{s}=1.751294$ and then decreasing down to $\approx 0.0021$ at the last sampled point at $r / r_{s}=2.01$. For a tangentially oriented atomic dipole, the frequency shift gradually increases from the value of $\approx-162$ at $r / r_{s}=1.005025$ up to $\approx-0.00017$ at $r / r_{s}=2.01$ and compares well with the quasi-static approximation [see Eq. (41)]. The latter predicts a monotonic increase of frequency shifts from large negative values to zero as $r / r_{s}$ increases. Had the sphere radius was larger compared to the emission wavelength, one would observe an oscillating relationship between the frequency shift and the position of a radiating atomic dipole for all dipole orientations and both inside and outside the sphere. In agreement with the quasi-static result (4) for a homogeneous dielectric sphere which is optically denser than surrounding medium $\left(\varepsilon_{s}>\varepsilon_{h}\right)$, the frequency shift of an atomic dipole inside the sphere and in close proximity to its boundary is always toward 
higher frequencies (blue shift) [13. On the other hand, the frequency of an atomic dipole outside the sphere and in close proximity to its boundary experiences a shift toward lower frequencies (red shift) [14].

It is clear from Figs. 2, 3 that frequency shifts of an atomic dipole interacting with the "nano-matryoshka" structures A-C experience significantly enhanced changes than in the comparative example of the homogeneous dielectric sphere $\mathbf{D}$. Already at the sphere center they can be more than two orders of magnitude larger: $\approx-1.941$ for $\mathbf{A}, \approx-1.691$ for $\mathbf{B}$, and $\approx 0.903$ for $\mathbf{C}$ compared to $\approx 0.0117$ for $\mathbf{D}$. The emission frequency of an atomic dipole in a silica region inside the "nano-matryoshka" structures $\mathbf{A}$ and $\mathbf{B}$ is always shifted toward lower frequencies (red shift). For "nano-matryoshka" structure $\mathbf{C}$, which radius is more than four times larger than that of $\mathbf{A}$ and $\mathbf{B}$, the red shift is still observed in a proximity of gold shells. However, in a marked contrast to "nano-matryoshka" structures $\mathbf{A}$ and $\mathbf{B}$, further away from gold shells, a small frequency shift toward higher frequencies is observed: for $r / r_{s} \in[0,0.17),(0.69,0.79)$ and $r / r_{s} \in[0,0.23),(0.7,0.85)$ in the case of tangentially and radially oriented dipole, respectively. The magnitude of frequency shifts substantially depends on the atom position within a dielectric shell. With the atom approaching metal shell boundaries, the frequencies exhibit an accelerated decrease toward large negative values. We have seen that even in the case of a purely homogeneous dielectric microsphere $\mathbf{D}$ with a small refractive index contrast, the frequency shifts are capable of reaching very high values near the surface of the microsphere. However, for the respective "nano-matryoshka" structures A-B, the shifts at the proximity of metaldielectric interfaces can be more than two orders of magnitude larger.

Outside and at a very close proximity of the outer sphere boundary, large red frequency shifts are observed for the complex nanoshells A-C as well as for the homogeneous spheres D-F. For instance, for radially oriented dipole source at the very first sampled point outside the spheres at $r / r_{s}=1.005025$, this red shift ranges from $-7392(\mathbf{B})$, through $-5858(\mathbf{F}),-5117(\mathbf{A}),-334(\mathbf{D})$, till $-67(\mathbf{C}, \mathbf{E})$. For a tangential dipole orientation, the red shift ranges from $-3597(\mathbf{B})$, through $-2839(\mathbf{F}),-2480(\mathbf{A}),-162(\mathbf{D})$, till -30 $(\mathbf{C}, \mathbf{E})$. Note that for the largest "nano-matryoshka" $\mathbf{C}$, and a homogeneous gold sphere $\mathbf{F}$ of the same radius, the comparable frequency shifts at the proximity of the outer sphere boundary are 5-times smaller than for the silica microsphere D. Surprisingly enough, the quasistatic result (44) of Klimov et al [13, 14] that, in the close proximity of a spherical shells interface, the radial frequency shift is approximately twice as large as the tangential frequency shift appears to apply also for complex nanoshells (see Figs. 2, 3).

Further away from sphere boundaries, as the value of $r / r_{s}$ increases, the red shift typically changes into blue one and vice versa in dampened oscillations around zero. According to Fig. 2, a noticeably large blue shift with maximum $\approx 2.14$ at $r / r_{s} \approx 1.154$ is observed for a radially oriented dipole source outside "nano-matryoshka" $\mathbf{B}$, leading to repulsive forces between the atom and the dielectric microsphere [14. The blue shift persists in a large interval for $r / r_{s} \in(1.12,1.78)$. For a tangential dipole source orientation, this blue shift, which occurs for $r / r_{s} \in(1.14,1.275)$ and $(1.63,2.01)$ with maximum $\approx 0.413$ at $r / r_{s} \approx 1.164$, becomes almost five-times smaller. Blue frequency shifts for the atom located outside a sphere are also observed in the remaining cases, but they are 
almost one order of magnitude smaller. For instance, for radially oriented dipole source, the excursion above zero does not exceeds $\approx 0.148$ for $\mathbf{C}\left(\right.$ at $\left.r / r_{s} \approx 1.144\right), \approx 0.146$ for $\mathbf{E}\left(\right.$ at $\left.r / r_{s} \approx 1.154\right), \approx 0.059$ for $\mathbf{A}\left(\right.$ at $\left.r / r_{s} \approx 1.592\right), \approx 0.033$ for $\mathbf{F}\left(\right.$ at $\left.r / r_{s} \approx 1.741\right)$, $\approx 0.004$ for $\mathbf{D}\left(\right.$ at $\left.r / r_{s} \approx 1.751\right)$. In the case of a tangentially oriented dipole source, the excursion above zero does not exceeds $\approx 0.113$ for $\mathbf{E}\left(\right.$ at $\left.r / r_{s} \approx 1.254\right), \approx 0.104$ for $\mathbf{C}$ (at $\left.r / r_{s} \approx 1.254\right), \approx 0.062$ for $\mathbf{A}\left(\right.$ at $\left.r / r_{s} \approx 2.01\right), \approx 0.05$ for $\mathbf{F}\left(\right.$ at $\left.r / r_{s} \approx 2.01\right)$, whereas for $\mathbf{D}$ the frequency shift remains negative till 2.01. Hence, similarly to the case of the atom located either close to a plane or inside a homogeneous dielectric spheres [13], the relationship between the frequency shift and the position of the atomic dipole outside the spheres considered here has an oscillating character The dampened oscillatory behaviour of the frequency shift for the radially oriented dipole outside the sphere $\mathbf{D}$ contradicts the conclusion reached by Klimov et al [14]. However, Klimov et al [14] have only studied atoms at a distance from a dielectric sphere not larger than $r / r_{s} \approx 1.2$, which is a too short distance to observe any oscillating behaviour. Had they drawn frequency shifts for larger values of $r / r_{s}$, they might have observed the oscillatory behaviour, too.

\subsection{Decay rates, the Ohmic loss contribution to nonradiative decay rates, and fluorescence yield}

\subsubsection{Total decay rate $W^{t}$}

The normalized total decay rates $W^{t}$ as calculated directly from the imaginary part of Green's function at coinciding arguments according to Eq. (5) (see also Eq. (135) of Ref. [12]), are displayed in Figs. 4, 5. Obviously, one finds the rates in the sphere center identical for the radial and tangential atomic dipole orientations. An advantage in dealing with the normalized decay rates is that any local-field correction [2, 3, 18, 36] cancels out (see also Sec. 4.3 below) and, in principle, a direct comparison between the normalized decay rates and experiment can be performed.

Similarly as in the preceding subsection, in order to appreciate changes in the decay rates induced by nanoshells $\mathbf{A}-\mathbf{C}$ geometry, we will first discuss the comparative example $\mathbf{D}$ of a homogeneous silica sphere. Inside the dielectric sphere $\mathbf{D}$, the normalized decay rate for a radially oriented atomic dipole steadily decreases from its maximum value of $\approx 0.94237$ at the sphere origin down to $\approx 0.83437$ at the last sampled point inside the sphere at $r / r_{s}=0.995075$. On the other hand, the normalized decay rate for a tangentially oriented atomic dipole exhibits a weakly oscillating behaviour: it first increases from the value of $\approx 0.94237$ at the sphere origin and reaches its maximum of $\approx 0.95173$ at $r / r_{s}=0.497562$, and then decreases down to $\approx 0.90179$ at $r / r_{s}=0.995075$. Outside the dielectric sphere $\mathbf{D}$, the normalized decay rate for a radially oriented atomic dipole steadily decreases from its maximum value of $\approx 1.27798$ at the first sampled point outside the sphere at $r / r_{s}=1.005025$ down to $\approx 0.99896$ at the last sampled point at $r / r_{s}=2.01$. On the other hand, the normalized decay rate for a tangentially oriented atomic dipole exhibits a weakly oscillating behaviour: it first increases from the value of $\approx 0.9824$ at $r / r_{s}=1.005025$, reaches its maximum of $\approx 1.00414$ at $r / r_{s}=1.860746$, and then it 
decreases down to $\approx 1.00366$ at the last sampled point outside the sphere at $r / r_{s}=2.01$. (Though due to the scale of ordinate axis, the decay rates appear to be a flat featureless horizontal line in Figs. 4, 5). Note in passing that for spheres of larger radius compared to the emission wavelength one would observe an oscillating behaviour of decay rates for any atomic dipole orientation [10, 11].

A characteristic feature of nanoshells is a huge increase of the decay rates for a dipole source in a close proximity to metal boundaries, and especially when dipole is within the silica core. In a purely dielectric case, such large decay rates are only observed in the proximity of sharp resonances of large spheres [10, whereas in the present case they can be achieved with small nanospheres without any special tuning to their internal resonances. For the "nano-matryoshka" structures $\mathbf{A}$ and $\mathbf{B}$, the decay rates monotonically increase from the values at their center of $\approx 0.8751$ and $\approx 1.7979$, respectively, up to the respective values at the last sampled core points of $\approx 2773(5412)$ and $\approx 2445$ (4765) for the tangential (radial) dipole orientation. The normalized decay rates of an atomic dipole at the core region of the largest "nano-matryoshka" $\mathbf{C}$ exhibit a qualitatively different behaviour which is characterized by pronounced minima $\approx 0.1696$ at $r / r_{s}=0.218955$ and $\approx 0.1129$ at $r / r_{s}=0.209005$ for the radial and tangential dipole orientations, respectively. Depending on the nanoshell parameters and the atom position, both inhibited and enhanced decay rates are observed, with the decay rates maximum values (in hot spots) being between two and three orders of magnitude larger than the decay rates minimum values (in cold spots) within the same shell. The positional sensitivity of the decay rates appears to be more pronounced in the nanoshell core regions than in the second silica shell.

Outside the complex nanoshell $\mathbf{C}$, an oscillatory dependence of the decay rates on the dipole position is clearly visible. The behaviour is closely matched by the case $\mathbf{E}$, i.e. the case of homogeneous metal sphere of the same radius. The amplitude of the oscillatory dependence is much stronger than that discussed earlier for D. A pronounced oscillatory dependence of the decay rates on the dipole position outside the sphere can also be seen for $\mathbf{F}$ and a tangential dipole orientation. For $\mathbf{A}$ and a tangential oriented dipole interacting with $\mathbf{B}$ only a very weak oscillatory dependence is seen. For $\mathbf{B}$ and a tangential dipole orientation and $\mathbf{F}$ and the radial dipole orientation only monotonic decrease of decay rates is observed down to $\approx 0.10373$ and $\approx 0.10188$, respectively, at $r / r_{s}=2.01$

\subsubsection{Radiative decay rate $W^{r a d}$}

The normalized radiative decay rates $W^{\text {rad }}$ as calculated according to Eq. (6) are shown in Figs. 6] and 7. The radiative decay rate is more pronounced for a radially oriented atomic dipole, in which case an order of magnitude enhancement can be expected for an optimal atom position inside the second silica shell of the nanoshells $\mathbf{A}$ and $\mathbf{B}$. Outside the spheres, the largest (an order of magnitude) enhancement of the radiative decay rate is achieved in a proximity of the small homogeneous gold microsphere $\mathbf{F}$, closely followed by the nanoshell $\mathbf{A}$. The best location of a tangentially oriented atomic dipole appears to be within the nanoshell $\mathbf{B}$ silica core. Whereas the radially oriented atomic dipole 
shows typically an enhanced radiative decay rate outside the spheres, in the case of its tangentially orientation the radiative decay rate is generally reduced. A strongly reduced radiative decay rate is also observed for an arbitrarily orientated atom inside the silica shells of the nano-matryoshka structure $\mathbf{C}$. Further away from the spheres outer surfaces the radiative decay rate shows dampened oscillations around one for any atomic dipole orientation. The radiative decay rate for a tangential dipole orientation exhibits a complex behaviour within the nanoshell $\mathbf{C}$ silica core, showing there a local minimum of $\approx 0.0204$ at $r / r_{s}=0.199055$.

\subsubsection{Ohmic loss contribution to the decay rate}

Compared to the normalized total and radiative decay rates in Figs. 4, 5, 6, and 7 , the normalized Ohmic loss contribution to the decay rates shown in Figs. 8 , 9 does not exhibit any oscillatory behaviour outside the spheres. It is reminded here that the Ohmic loss contribution to the decay rates has been calculated according to Eqs. (77), (8). (Further computational details can be found in Secs. 6 and 8.2 of Ref. [12.) In agreement with expectations, the Ohmic loss contribution steadily decreases down to zero with the increased atomic dipole distance from the sphere surface. It is also easily understandable that the Ohmic loss contribution to the decay rates rapidly increases when the atom approaches metal boundary. However, rather contra-intuitively, the Ohmic loss contribution within the nanoshell $\mathbf{C}$ silica core decreases from its maximum value of $\approx 0.2102$ at the center down to $\approx 0.044$ and $\approx 0.047$ for the radial and tangential dipole orientations, respectively, at the very last sampled silica core point at $r / r_{s}=0.567214$. For the radial dipole orientation, the Ohmic loss contribution decreases monotonically, whereas for a tangential dipole orientation it exhibits an oscillatory dependence with a series of local minima and maxima: first decreasing from the central value of $\approx 0.2102$ down to $\approx 0.086$ at $r / r_{s}=0.228905$, increasing up to $\approx 0.137$ at $r / r_{s}=0.398060$, and eventually decreasing down to $\approx 0.047$ at $r / r_{s}=0.567214$. This effect appears to be real and not an artifact of computational inaccuracies or numerical instabilities. Calculations in extended precision yielded essentially the same result. Note that the local minima of the decay rates and the Ohmic loss contribution to the decay rate for a tangential dipole orientation occur at more or less the same position within the nanoshell $\mathbf{C}$ silica core.

\subsubsection{Fluorescence yield}

In Fig. 10 the ratio $W^{r a d} / W^{t}$ is plotted, which is known as the fluorescence yield, or simply quantum efficiency. Our $W^{t}$ is that calculated directly from the imaginary part of Green's function at coinciding arguments according to Eq. (15) and has earlier been shown in Figs. 4, 5. However, our $W^{t}$ only comprises the Ohmic loss contribution, $W_{\Omega}^{n r a d}$, to the nonradiative decay rate $W^{n r a d}$. The Ohmic loss is only one of many nonradiative mechanisms, such as, for instance, multiphoton relaxation, coupling to defects, direct electron-transfer processes, and concentration quenching, which all contribute to the nonradiative decay rate $W^{\text {nrad }}$ [39, 40, 41, 42, 43] and which may all occur at real experimental

situations. Therefore, our $W^{t}=W^{\text {rad }}+W_{\Omega}^{\text {nrad }}$ does only provide the lower bound for the 
total decay rate $W^{\text {tot }}$. Since $W^{\text {rad }} / W^{\text {tot }} \leq W^{\text {rad }} /\left(W^{\text {rad }}+W_{\Omega}^{\text {nrad }}\right)$, the ratio $W^{\text {rad }} / W^{t}$ for an averaged dipole orientation plotted in Fig. 10 provides an upper theoretical bound on the fluorescence yield. Because of absorbing bodies, $W_{\Omega}^{n r a d}>0$ and the respective ratios $W^{\mathrm{rad}} / W^{t}$ are always smaller than one. (For the sake of clarity, the trivial non-absorbing case $\mathbf{D}$, in which case the ratio is equal to unity for any atom position, has been omitted.) As expected, with increasing the atom distance from the outer sphere surface, where the Ohmic loss contribution $W_{\Omega}^{\text {nrad }}$ decreases to zero, the ratio $W^{\text {rad }} / W^{t}$ rapidly approaches unity. For all cases considered, the respective fluorescence yields are already larger than 0.93 at $r / r_{s}=2$. At nanoshell centers, a remarkably large value of the fluorescence yield $(\approx 0.694)$ is observed for nanoshell $\mathbf{B}$. In the case of nanoshell $\mathbf{C}$, the fluorescence yield is the smallest $(\approx 0.160)$. As dipole approaches metallic shells, the respective fluorescence yields rapidly drop to very small values. This is well-known as fluorescence quenching [4. In spite of decay rates being strongly enhanced in the proximity of metal shells (see Figs. 4. 5), the decay of the excited atomic states is not accompanied by the emission of a real photon, but instead matter quanta are created due to absorption. The fluorescence quenching at the proximity of metal boundaries then implies pronounced maxima of the respective fluorescence yields when the atom is located at the middle of the second silica shell of nano-matryoshka structures A-C. Indeed, the shell is surrounded by metal shells on its both sides. A noticeable feature is also a rather complex behaviour of the fluorescence yield within the core of nanoshell $\mathbf{C}$. In the latter case, we have already seen a complex behaviour in the case of decay rates and the Ohmic loss contribution to the decay rates. The complex behaviour in all these cases is the result of almost $5 \times$ larger core radius compared to the core radii of nanoshells $\mathbf{A}$ and $\mathbf{B}$.

\subsection{Photobleaching and detected intensity enhancement}

If one assumes that photobleaching of a dye takes place only while the dye is in its excited states, a sufficiently large enhancement of the spontaneous emission rates can significantly lower the probability of switching into nonfluorescent dark (triplet) states, thereby increasing stability against photobleaching [32, 50. The latter means that a fluorescent dye molecule can emit more photons before irreversible chemical reactions prevent the molecule from any further emission. Let us assume that photostability is inversely proportional to the excited state lifetime, i.e., proportional to the total decay rate [20]. Then the photostability of the atom interacting with a nanoshell compared to that in the free-space filled in with a homogeneous medium which is identical to that at the atom position increases by the factor of $W^{\text {tot }} / W_{h}^{\text {rad }}$. On the other hand, there is only the probability of $W^{r a d} / W^{\text {tot }}$ that a given decay will end up with photon at the spatial infinity. Given the respective two situations, let $N$ and $N_{0}$ be average numbers of the photons emitted by the fluorescence source (and detected in the far-field) till photobleaching. Then the ratio $N / N_{0}$ can be obtained as $\left(W^{\text {tot }} / W_{h}^{r a d}\right) \times\left(W^{r a d} / W^{\text {tot }}\right)=W^{r a d} / W_{h}^{r a d}$, i.e., the ratio is equal to the normalized radiative decay rate. The latter has been plotted as a function of the source position in Figs. [6] and 7.

The results presented so far have not assumed any incident radiation. An atomic 
dipole has already been assumed to be in an excited state and the changes in its decay properties have been monitored. It is informative to assess the changes in the detected fluorescence intensity due to the presence of a metal nanoshell. By taking into account that

1. the respective stimulated emission and absorption probabilities undergo the same changes in the presence of material boundaries as the rate of the spontaneous emission;

2. the stimulated emission and absorption rates are given as the product of the intensity, or squared field strength, with the respective probabilities;

3. the spontaneous decay rate $W^{t}$ can be enhanced by more than 3 orders of magnitude (see Figs. 4, 5), the radiative decay rate $W^{\text {rad }}$ an order of magnitude (see Figs. 6], 7), and intensity by 4 or more orders of magnitude;

one finds that an elementary absorption-emission cycle can be accelerated by 7 or more orders of magnitude, resulting in the enhancement by 5 or more orders of magnitude of the detected fluorescence intensity.

\section{Discussion}

\subsection{Size-dependent corrections and nonlocal effects}

The calculations presented so far have been performed assuming the bulk values of gold dielectric constant. If theory presented here is to be applied for a multilayered spherical particle with a small metallic core or a thin metallic shell with a radius or thickness $S \lesssim 20$ $\mathrm{nm}$ (such as the outer shell of nano-matryoshka B), two effects have to be additionally considered. First, the bulk dielectric function is modified, since the electronic mean free path is then shorter than in the bulk 45. Second, nonlocal effects come into play [46, 47, 48. The first effect can easily be incorporated by replacing the bulk dielectric function $\varepsilon_{B}(\omega)$ with its size-dependent modification

$$
\varepsilon(\omega)=\varepsilon_{B}(\omega)+\frac{\omega_{p}^{2}}{\omega^{2}+i \omega \tau_{B}^{-1}}-\frac{\omega_{p}^{2}}{\omega^{2}+i \omega \tau^{-1}} .
$$

Here $\omega_{p}$ is the bulk plasmon frequency, $\tau_{B}$ is the relaxation time in the bulk metal, $\tau^{-1}=\tau_{B}^{-1}+v_{F} S^{-1}$ is the inverse relaxation time (also called damping coefficient $\Gamma$ ) corrected for the finite size of the particle, and $v_{F}$ is the Fermi velocity. More generally,

$$
\tau^{-1}=\tau_{B}^{-1}+A v_{F} S^{-1}
$$

where $A$ is a parameter determined by the geometry. For simple Drude theory and isotropic scattering one usually takes $A=1$.

On the other hand, nonlocal effects, i.e., when the Fourier transform of the dielectric function depends in addition to $\omega$ also on $\mathbf{k}$, are associated with the resonant excitation 
of longitudinal bulk plasmon modes (either propagating ones, with frequency above the plasma frequency $\omega_{p}$, or evanescent ones, with frequency below the plasma frequency $\omega_{p}$ ) 46. 47, 48. The neglect of the nonlocal responses of the substrate is the main reason why a phenomenological treatment will generally break down when the radiating atom is very close (within a few nanometers) to the sphere surface [48. Indeed, the spectroscopic properties of the two-level atom interacting with a complex spherical nanoshell have been investigated here within the framework of macroscopic Maxwell's equations. The latter lead to unphysical results in the limit $r \rightarrow r_{s}$, i.e., when the atom approaches sphere surface. For instance, the quasi-static approximation [see Eq. (41)] predicts that frequency shifts increase as $\left(r-r_{s}\right)^{-3}$. The non locality brings about a natural cutoff pole order: the nonlocal sphere does not polarize significantly at angular momenta higher than a certain cutoff value $l_{c}$. The latter is of principal importance since it allows for a fully converged treatment of multipolar excitation effects [49]. Another important feature of the nonlocal dielectric function is that it introduces a natural cutoff angular momentum for the excitation of near-field modes [48, 49], and thereby a natural cutoff for the convergence of the nonradiative decay rates.

The nonlocal effects may significantly influence both the radiative and nonradiative rates of very small nanospheres and nano-matryoshka structures with very thin shells. For instance, in the case of a small homogeneous nanosphere with radius of $r_{s} \leq 5 \mathrm{~nm}$, the radial and tangential dipolar decay rates for low emission frequencies $\left(\omega \leq 0.5 \omega_{p}\right)$ can be reduced by up to 2 orders of magnitude with respect to the local results [48. On the other hand, for emission frequencies $\omega \geq 0.5 \omega_{p}$, the decay rates can be up to 2 orders of magnitude larger than in the local case [48. The theory of Leung [48] has been extended to the case of complex nanoshells (see Sec. 11.1 of Ref. [12) and its application will be presented elsewhere.

\subsection{Radiative decay rates vs the local density of states}

In the ideal case of nonabsorbing and nondispersive material medium (which, however (except for the vacuum), does not exist), the trace of the imaginary part of the Green's function at coinciding spatial arguments, which enters Eq. (5), is related to the local density of states, $\rho(\omega, \mathbf{r})=-(1 / \pi) \operatorname{Tr} \operatorname{Im} \mathbf{G}\left(\mathbf{r}_{d}, \mathbf{r}_{d}, \omega_{0}\right)$, where $\operatorname{Tr}$ denotes trace $(\mathbf{G}$ is a tensor quantity). This relation has prompted claims that radiative decay rates are proportional to the local density of states (LDOS). However, even in the ideal nonabsorbing homogeneous case one finds this claim true only when (i) one performs orientational averaging over atomic dipole orientations and (ii) properly includes medium dependent prefactors [see Eq. (5)]. Once dispersion comes into play, radiative decay rates cease to be proportional to the LDOS. Indeed, Nienhuis and Alkemade [37] have showed that the LDOS in the homogeneous dispersive case is

$$
\rho(\omega)=n^{2} \frac{d(\omega n)}{d \omega} \rho^{(0)}(\omega)
$$

where $n(\omega)=\sqrt{\varepsilon \mu}$ is the frequency dependent refractive index of the medium and $\rho^{(0)}(\omega)=\omega^{2} / c^{3} \pi^{2}$ is the LDOS of photon states in vacuum (see Eqs. (29-31) of Ref. 
[37]). Yet, as it has been emphasized, the Nienhuis and Alkemade formula (11), which does not contain any derivatives of the medium refractive index, remains also to be valid in the dispersive case.

The presence of a derivative factor in the LDOS can also be traced down to similar derivative factors in the Brillouin expression for the electromagnetic field energy density (9). Another argument is as follows. Let $G(E)=1 /(E-H)$ denote a scattering Green's function, where $E$ is an energy and $H$ is a Hamiltonian. Then, for an isolated eigenvalue $E_{n}$ of an energy dependent Hamiltonian (our case; see Refs. [7, 8]) the quantity $-(1 / \pi) \operatorname{Tr} \operatorname{Im} G(E)$ is no longer equal to the Dirac delta function $\delta\left(E-E_{n}\right)$, but merely proportional to $\delta\left(E-E_{n}\right)$ with a prefactor $1 /|1-d H / d E|$. The latter is, in general, different from unity. Therefore, the density of states (DOS) $\rho(E)$ can no longer be defined as $\rho(E) \equiv-(1 / \pi) \operatorname{Tr} \operatorname{Im} G(E)$. Yet another argument is that the integrated density of states is given by the expression $N(E) \equiv(1 / \pi) \operatorname{Tr} \operatorname{Im} \ln G(E)=-(1 / \pi) \operatorname{Tr} \operatorname{Im} \ln (E-H)$. Since, for an energy dependent Hamiltonian, the relation $(d / d E)[\operatorname{Tr} \operatorname{Im} \ln G(E)]=-\operatorname{Tr} \operatorname{Im} G(E)$

no longer holds, $N(E) \neq \int^{E} \rho(E) d E$, provided that the density of states (DOS) $\rho(E)$ is defined as $\rho(E) \equiv-(1 / \pi) \operatorname{Tr} \operatorname{Im} G(E)$ (see also Ref. [51]).

\subsection{Local-field corrections}

An atomic dipole couples to the microscopic vacuum fluctuations. If the (unnormalized) decay rate $W^{t}$ [see Eq. (5)] is obtained from macroscopic Maxwell's equation, the difference between microscopic and macroscopic vacuum fluctuations can be accounted for by local-field corrections. The local field effects always play an important role and constitute a critical test of our understanding of the relation between microscopic and macroscopic electromagnetic phenomena [2, 3]. As discussed by Schuurmans et al [36], one has to distinguish between the substitutional and interstitial character of impurities. In the former case the well-known empty-cavity factor $3 \varepsilon /(2 \varepsilon+1)$ applies, whereas, in the latter case, the Lorentz local-field factor $(\varepsilon+2) / 3$ is obtained. Since the decay rate $W^{t}$ can be expressed in terms of an expectation value of the product of two electric field operators (see, e.g., Eq. (25) of Ref. [8]), the local-field factors appear squared. In the near-infrared and for optical wavelengths, an inclusion of the corresponding local-field factor is only necessary for electric dipole transitions. Indeed, in the above wavelength range, magnetic permeabilities $\mu$ of different materials are all equal to the vacuum value and, therefore, the local-field factors for magnetic dipole transitions are trivially equal to one. (For local-field corrections in absorbing case see Ref. [18.)

However, since we have only discussed normalized decay rate $W^{t}[$ see Eq. (5)], any local-field correction cancels out and a direct comparison between the normalized decay rates and experiment can, in principle, be performed (see, however, the following subsections). 


\subsection{Nonradiative decay rates}

Earlier, at the end of Sec. 3.2 it has been discussed that the sum $W^{t}=W^{\mathrm{rad}}+W_{\Omega}^{\text {nrad }}$, where $W_{\Omega}^{\text {nrad }}$ stands for the nonradiative decay rate due to the Ohmic losses, does only provide the lower bound on the total decay rate $W^{\text {tot }}$. Consequently, Fig. 10 only shows the upper theoretical limits on the fluorescence yields. Indeed, the Ohmic loss is only one of many other nonradiative mechanisms, such as, for instance, multiphoton relaxation, coupling to defects, direct electron-transfer processes, and concentration quenching, which all contribute to the nonradiative decay rate $W^{\text {nrad }}$ [39, 40, 41, 42, 43]. It turns out that even in a purely dielectric case, in the absence of any Ohmic losses and for small fluorescence atom concentrations, the nonradiative decay $W^{\text {nrad }}$ can be higher than the radiative decay $W^{\mathrm{rad}}$, resulting in the fluorescence yield smaller than 0.5 [3, 52, 53. When fluorescence dye concentrations increase above a certain threshold value, the fluorescence yields of most organic dyes are substantially reduced even further with respect to a zero-concentration limit value. It should be emphasized that, for a dipole outside the sphere, the radiative decay rate $W^{\text {rad }}$ is proportional to the intensity of the time-resolved fluorescence spectra at $t=0$ 43. Therefore, in the latter case, $W^{\text {rad }}$ and $W^{\text {nrad }}$ can be disentangled experimentally 43 .

In order to test theoretical predictions experimentally, the choice of a suitable fluorescence source turns out to be a critical issue. Rare-earth ions, which exhibit long luminescence lifetimes, are very suitable candidates [2, 42, 54]. However, they are usually implanted by an ion deposition resulting in a poor control over their radial distribution within a spherical shell. On the other hand, fluorescent organic groups can be placed inside the dielectric core or shell of a complex nanoparticle with nanometer control over the radial position [26], but their decay rates are usually strongly affected also by other then purely electromagnetic mechanisms, such as concentration quenching. For instance, in the case of fluorescein (FITC), the threshold value for a concentration quenching is $\sim 0.1$ $\mathrm{mM}(\sim 1 \mathrm{mM})$ in liquid (solid) solutions [50]. However pyrene-doped PMMA spheres with pyrene concentrations up to $10 \mathrm{mM}$ do not exhibit any concentration dependence [55]. Note also that metals per se have inherent photoluminescence [57]. However, since metal photoluminescence results in a broad band continuum [35, 57], it can easily be filtered out.

\subsection{Nonlocal decay rates}

The nonradiative mechanisms different from the Ohmic losses do only depend on the immediate neighborhood of a radiating dipole. Therefore, one can include all such mechanisms of nonradiative decay rates under the local nonradiative decay rate, $W^{\text {loc }}$. On the other hand, the nonradiative decay rate due to Ohmic losses and the radiative decay rate can be viewed as a nonlocal decay rate, $W^{\text {nloc }} \equiv W^{t}=W^{\text {rad }}+W_{\Omega}^{\text {nrad }}$. The reason behind this nomenclature is that the latter two rates depend on the geometry and material composition of the entire sphere and surrounding medium, and not only on the immediate proximity of the radiating dipole. The total decay rate is then written as 
$W^{\text {tot }}=W^{l o c}+W^{\text {nloc }}$. As a rule, both the nonradiative decay rate due to Ohmic losses and the radiative decay rate change if the optical properties of a shell being far away from the radiating dipole (for instance, ambient medium) change [42, 52, 53. In the case of a homogeneous dielectric sphere, the local and nonlocal decay rates can be, in principle, disentangled by measuring the total decay rate $W^{\text {tot }}$ using the same sphere in different environments [2, 3, 52. For instance, the sphere can be embedded in a refractive index matched liquid [2, 3, 52]. Its radiative decay rates then becomes that of a dipole in a homogeneous dielectric slab [53, 56. Other possible environments include air or liquids with different refractive indices [52]. The difference of the respective total decay rates measured in different sphere environments then corresponds to the difference of the nonlocal decay rates [52. The latter is obviously also true for a multicoated sphere. The local and nonlocal decay rates can then be separated by fit of Eq. $W^{t o t}=W^{l o c}+W^{\text {nloc }}$ to the measured data [42, 52. Only after the local decay rate $W^{l o c}$ is determined, a comparison of measured data and theory presented here can be performed 32 .

\subsection{Numerical subtleties}

When dealing with dispersive and absorbing shells, it may happen that the linearly independent spherical Bessel functions $j_{l}$ and $y_{l}$ (see Sec. 10 of Ref. [58 for notations) are in fact related by $y_{l}(k r) \approx i j_{l}(k r)$ up to almost all significant digits in double precision. Consequently, if the spherical Hankel functions $h_{l}^{(1)}$ is later on formed as the sum $h_{l}^{(1)}(k r)=j_{l}(k r)+i y_{l}(k r)$ [58, its precision may be drastically compromised. Therefore, it is always recommended to determine $h_{l}^{(1)}(k r)$ by a direct independent recurrence, such as that proposed by Mackowski et al [59] [see recurrences (63),(64) therein]. Otherwise the radiative decay result for the interior of the nanoshell $\mathbf{C}$ may differ by up to four-orders of magnitude from the correct one. If one can perform calculations in an extended precision, this pathological behaviour can be largely overcome, yet the independent recurrence by Mackowski et al [59] is still highly recommended.

\subsection{Outlook}

Interesting avenue of further research is the study of the effect of sphere's resonances on the atomic spectroscopic properties. So far, the latter problem has been thoroughly investigated only in the case of a homogeneous dielectric sphere [14 and a dispersive and absorbing sphere [8]. However, a comparative study for complex nanoshells is missing. Yet another interesting set of problems arises in connection with strong atom-sphere interactions, leading, for instance, to Rabi splitting of an atom levels. Also here only the case of a dielectric homogeneous microsphere has been considered 60.

Another important aspect is the inclusion of nonlocal effects into the treatment of spectroscopic properties of the atom interacting with complex nanoshells. As it has been discussed earlier, the neglect of the nonlocal responses of the substrate is the main reason why a phenomenological treatment will generally break down when the radiating atom is very close (within a few nanometers) to the sphere surface [48. So far, the effect 
has only been investigated in the case of small metal nanospheres [48. In this regard, complex "nano-matryoshka" structures offer the possibility in studying the nonlocal effects for spheres of relatively large radius, provided that metal shells are thin enough. General theory for the case of "nonlocal" shells has already been developed [12] and its application will be presented elsewhere.

\section{$5 \quad$ Summary and conclusions}

Frequency shifts, decay rates, the Ohmic loss contribution to the nonradiative decay rates, fluorescence yields, and photobleaching of an atomic dipole interacting with "nanomatryoshka" structures of Prodan et al [22] have been investigated. The changes in the spectroscopic properties of an atomic dipole interacting with complex nanoshells have been shown to be significantly enhanced, often more than two orders of magnitude, compared to the same atom interacting with a homogeneous dielectric sphere. The detected fluorescence intensity can be then enhanced by 5 or more orders of magnitude. The decay rate enhancements can be achieved with small nanospheres without any special tuning to their internal resonances. The changes strongly depend on the nanoshell parameters and the atom position. Rather contra-intuitively the Ohmic loss contribution to the nonradiative decay rates for an atomic dipole within the silica core of larger nanoshells may be decreasing when the silica core - inner gold shell interface is approached. Surprisingly enough, the quasistatic result (4) of Klimov et al [13, 14] that the radial frequency shift in the close proximity of a spherical shell interface is approximately twice as large as the tangential frequency shift appears to also apply for complex nanoshells (see Figs. 2, 3). Although decay rates are strongly enhanced in the proximity of metal shells, the majority of the emitted radiation is absorbed and fluorescence yield exhibits there a well-known quenching (see Fig. 10). Although simulation have so far been performed at the wavelength of 595 $\mathrm{nm}$ (the emission wavelength of lissamine molecules [43]), Fig. 11] demonstrates that significantly modified spectroscopic properties can be observed in a broad band comprising all (nonresonant) optical and near-infrared wavelengths.

It has been shown here that complex nanoshell structures provide the possibility of a controlled tunability in engineering of the radiative decay rates. The ability of controlled modification of radiative rates for atoms or molecules in the excited state is of great importance since dissipative pathways of the excited state can be controlled. For instance, one can design nanoprobes with enhanced quantum yield for fluorescent microscopy and with enhanced photostability for for biophysical and biomedical applications [4, 32, identification of biological particles in fluorescence-activated flow of cytomeres [61], to monitor specific cell functions, or in the cell identification and sorting systems [62, 63. Enhanced spontaneous emission rates could also provide increased sensitivity in low level fluorescence applications [40, 50]. Designing of small noble metal nanoparticles with reduced quantum yield, $W^{\text {rad }} / W^{\text {tot }}$, at the particle close proximity (cca $1 \mathrm{~nm}$ ) may have crucial implications for the particles use as acceptors in biophysical Förster resonant energy transfer experiments in vitro as well as in vivo [43. The theory presented earlier in Ref. [12] 
with numerical results shown here may also stimulate design of coated tip geometries for applications in near-field optical microscopy [35].

The emphasis was on the spontaneous decay, rates. However, detailed balance in thermal equilibrium implies that the knowledge of a single Einstein coefficient is sufficient to determine the remaining two. Therefore, qualitatively similar behaviour as that shown in Figs. 4, 5is also expected for the stimulated emission and absorption rates.

Hopefully, the results presented in this article, in conjunction with computer program freely available at http://www.wave-scattering.com, will provide a larger freedom in engineering of (complex) spherical particles properties, rendering them more suitable for a variety of applications. 


\section{References}

[1] E. M. Purcell, Phys. Rev. 69, 681 (1946); K. H. Drexhage, J. Lumin. 1-2, 693 (1970); D. Kleppner, Phys. Rev. Lett. 47, 233 (1981).

[2] G. L. J. A. Rikken and Y. A. R. R. Kessener, Phys. Rev. Lett. 74, 880 (1995).

[3] P. Lavallard, M. Rosenbauer, and T. Gacoin, Phys. Rev. A 54, 5450 (1996).

[4] J. R. Lakowicz, Anal. Biochem. 298, 1 (2001).

[5] G. S. Agarwal, Phys. Rev. A 12, 1475 (1975).

[6] J. M. Wylie and J. E. Sipe, Phys. Rev. A 30, 1185 (1984).

[7] H. T. Dung, L. Knöll, and D.-G. Welsch, Phys. Rev. A 62, 053804 (2000).

[8] H. T. Dung, L. Knöll, and D.-G. Welsch, Phys. Rev. A 64, 013804 (2001).

[9] R. R. Chance, A. Prock, and R. Silbey, Phys. Rev. A 12, 1448 (1975).

[10] H. Chew, J. Chem. Phys. 87, 1355 (1987).

[11] H. Chew, Phys. Rev. A 38, 3410 (1988).

[12] A. Moroz, Ann. Phys. (NY) 315, 352-418 (2005) (has been available on-line since October 11, 2004).

[13] V. V. Klimov, M. Ducloy, and V. S. Letokhov, J. Mod. Opt. 43, 549 (1996).

[14] V. V. Klimov, M. Ducloy, and V. S. Letokhov, J. Mod. Opt. 43, 2251 (1996).

[15] R. Ruppin, J. Chem. Phys. 76, 1681 (1982).

[16] H. Chew, M. Kerker, and P. J. McNulty, J. Opt. Soc. Am. 66, 440-444 (1976).

[17] K. G. Sullivan and D. G. Hall, Phys. Rev. A 50, 2708 (1994).

[18] M. S. Tomaš, Phys. Rev. A 63, 053811 (2001).

[19] V. V. Klimov and V. S. Letokhova, Chem. Phys. Lett. 301, 441 (1999).

[20] J. Enderlein, Appl. Phys. Lett. 80, 315 (2002).

[21] L.-W. Li, P.-S. Kooi, M.-S. Leong, and T.-S. Yee, IEEE Trans. Microwave Theory Techn. 42, 2302 (1994).

[22] E. Prodan, C. Radloff, N. J. Halas, and P. Nordlander, Science 302, 419 (2003).

[23] L. M. Liz-Marzán, M. Giersig, and P. Mulvaney, Langmuir 12, 4329 (1996). 
[24] T. Ung, L. M. Liz-Marzán, and P. Mulvaney, Langmuir 14, 3740 (1998).

[25] V. V. Hardikar and E. J. Matijević, J. Colloid Interface Sci. 221, 133 (2000).

[26] A. van Blaaderen and A. Vrij, Langmuir 8, 2921 (1992).

[27] K. P. Velikov, A. Moroz, and A. van Blaaderen, Appl. Phys. Lett. 80, 49 (2002).

[28] S. J. Oldenburg, R. D. Averitt, S. L. Westcott, and N. J. Halas, Chem. Phys. Lett. 288, 243 (1998).

[29] S. J. Oldenburg, J. B. Jackson, S. L. Westcott, and N. J. Halas, Appl. Phys. Lett. 75, 2897 (1999).

[30] J. B. Jackson and N. J. Halas, J. Phys. Chem. B 105, 2743 (2001).

[31] C. Graf and A. van Blaaderen, Langmuir 18, 524 (2002).

[32] C. Graf, D. J. van den Heuvel, A. Moroz, H. C. Gerritsen, and A. van Blaaderen, "Enhanced photostability and reduced lifetimes of dye molecules in colloidal gold shell particles", in preparation.

[33] A. E. Neeves and M. H. Birnboim, J. Opt. Soc. Am. B 6, 787 (1989).

[34] J. B. Jackson, S. L. Westcott, L. R. Hirsch, J. L. West, and N. J. Halas, Appl. Phys. Lett. 82, 257 (2003).

[35] A. Bouhelier, M. R. Beversluis, and L. Novotny, Ultramicroscopy 100, 413 (2004).

[36] F.J.P. Schuurmans, P. de Vries, and A. Lagendijk, Phys. Lett. A 264, 472 (2000).

[37] G. Nienhuis and C. Th. J. Alkemade, Physica C 81, 181 (1976).

[38] A. Tip, Phys. Rev. A 57, 4818 (1998).

[39] A. J. Campillo, J. D. Eversole, and H.-B. Lin, Phys. Rev. Lett. 67, 437 (1991).

[40] P. B. Bisht, K. Fukuda, and S. Hirayama, J. Chem. Phys. 105, 9349 (1996).

[41] B. I. Ipe, K. G. Thomas, S. Barazzouk, S. Hotchandani, and P. V. Kamat, J. Phys. Chem. B 106, 18 (2002).

[42] M. J. A. de Dood, L. H. Slooff, A. Moroz, A. Polman, and A. van Blaaderen, Appl. Phys. Lett. 79, 3585 (2001).

[43] E. Dulkeith et al, Phys. Rev. Lett. 89, 203002 (2002).

[44] Handbook of Optical Constants of Solids, edited by E. D. Palik (Academic, New York, 1985). 
[45] U. Kreibig and L. Genzel, Surf. Sci. 156, 678 (1985).

[46] A. R. Melnyk and M. J. Harrison, Phys. Rev. B 2, 835 (1970).

[47] R. Ruppin, Phys. Rev. B 11, 2871 (1975).

[48] P. T. Leung, Phys. Rev. B 42, 7622 (1990).

[49] R. Fuchs and F. Claro, Phys. Rev. B 35, 3722 (1987).

[50] A. Imhof, M. Megens, J. J. Engelberts, D. T. N. de Lang, R. Sprik, and W. L. Vos, J. Phys. Chem. B 103, 1408-1415 (1999).

[51] A. Moroz, Single dispersive impurity in a photonic crystal, in Proceeding of the Academic Colloquium "Quantum Optics of Small Structures", D. Lenstra, T. D. Visser, and K. A. H. Leeuwen eds. (The Royal Netherlands Academy of Arts and Sciences, Amsterdam, 2000), pp. 29-32 (preprint available at http://www.wave-scattering.com/impl.pdf).

[52] M. J. A. de Dood, L. H. Slooff, A. Moroz, A. Polman, and A. van Blaaderen, Phys. Rev. A 64, 033807 (2001).

[53] E. Snoeks, A. Lagendijk, and A. Polman, Phys. Rev. Lett. 74, 2459 (1995).

[54] H. Schniepp and V. Sandoghdar, Phys. Rev. Lett. 89, 257403 (2002).

[55] H. Fujiwara, K. Sasaki, and H. Masuhara, J. Appl. Phys. 85, 2052 (1999).

[56] H. P. Urbach and G. L. J. A. Rikken, Phys. Rev. A 57, 3913 (1998).

[57] A. Mooradian, Phys. Rev. Lett. 22, 185 (1969).

[58] M. Abramowitch and I. A. Stegun, Handbook of Mathematical Functions (Dover Publications, 1973).

[59] D. W. Mackowski, R. A. Altenkirch, and M. P. Menguc, Appl. Opt. 29, 1551 (1990).

[60] V. V. Klimov, M. Ducloy, and V. S. Letokhov, J. Mod. Opt. 44, 1081 (1997).

[61] B. H. Mayall and B. L. Gledhill, J. Histochem. Cytochem. 27, 1 (1979).

[62] P. F. Mullaney and P. N. Dean, Biophys. J. 10, 764 (1970).

[63] X. Yataganas and B. D. Clarkson, J. Histochem. Cytochem. 22, 651 (1974). 


\section{Figure captions}

Figure 1 - A typical spherical complex nanoshell, or "nano-matryoshka", and its parameters. In the present case, the "nano-matryoshka" will be embedded in water and the respective shaded and unshaded "nano-matryoshka" concentric regions will represent gold and silica shells.

Figure 2 - The normalized level shifts for the radially oriented atomic dipole radiating at wavelength of $595 \mathrm{~nm}$ as a function of its distance from the sphere center. The shifts are normalized to the radiative decay rate $W_{h}^{\text {rad }}$ in free-space filled in with the medium at the dipole position. For the homogeneous dielectric sphere $\mathbf{D}$, the frequency shift reaches the value as large as 261 at $r / r_{s}=0.995075$. However, for a better view of the region around zero frequency shift, the ordinate axis has been terminated at the frequency shift of 10 .

Figure 3 - The same as in Fig. 2 but for tangentially oriented atomic dipole. For the homogeneous dielectric sphere $\mathbf{D}$, the frequency shift reaches the value as large as 133 at $r / r_{s}=0.995075$. However, as in Fig. 2, for a better view of the region around zero frequency shift, the ordinate axis has been terminated at the frequency shift of 10 .

Figure 4 - Normalized decay rates $W^{t} / W_{h}^{\text {rad }}$ for the radially oriented atomic dipole radiating at wavelength of $595 \mathrm{~nm}$ as a function of its distance from the sphere center. The rates are normalized to the radiative decay rate $W_{h}^{\text {rad }}$ of the same atomic dipole in the free-space filled in with the medium at the dipole position.

Figure 5 - The same as in Fig. 4 but for tangentially oriented atomic dipole.

Figure 6 - The normalized radiative decay rate of the radially oriented fluorescence dipole source at wavelength of $595 \mathrm{~nm}$ The rate has been normalized to that in free-space filled in with the medium at the source position.

Figure 7 - The same as in Fig. [6 but for tangentially oriented atomic dipole.

Figure 8 - The normalized Ohmic loss contribution $W_{\Omega}^{\text {nrad }} / W_{h}^{\text {rad }}$ to the nonradiative decay rates for radially oriented atomic dipole radiating at wavelength of $595 \mathrm{~nm}$ as a function of its distance from the sphere center. The Ohmic loss contribution has been normalized to the free-space radiative decay rate $W_{h}^{r a d}$ in the medium identical to that at the dipole position.

Figure 9 - The same as in Fig. 8 but for tangentially oriented atomic dipole.

Figure 10 - Fluorescence yield (quantum efficiency) at wavelength of $595 \mathrm{~nm}$ for an averaged dipole orientation as a function of the dipole position.

Figure 11 - Normalized decay rates $W^{t} / W_{h}^{r a d}$ for an averaged dipole orientation in the case of nanoshell $\mathbf{A}$ as a function of the dipole position for different emission wavelengths. 


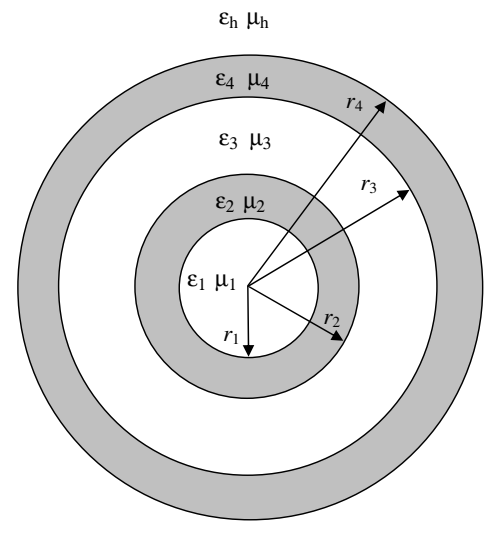

Figure 1: 


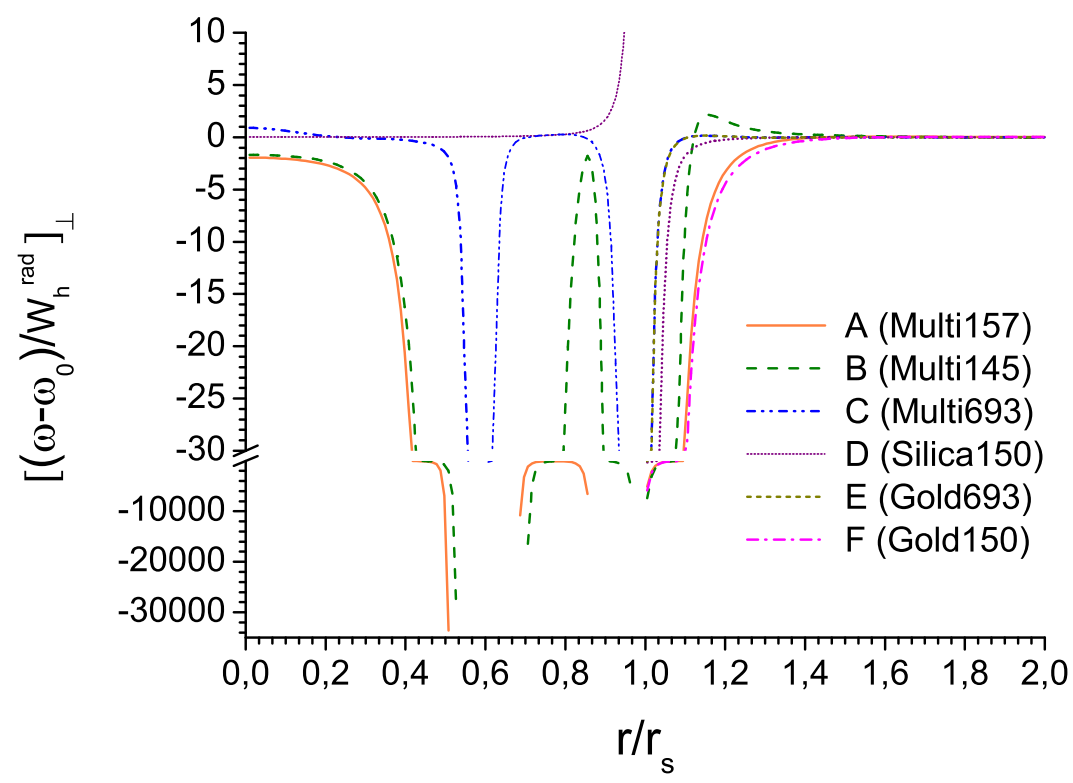

Figure 2:

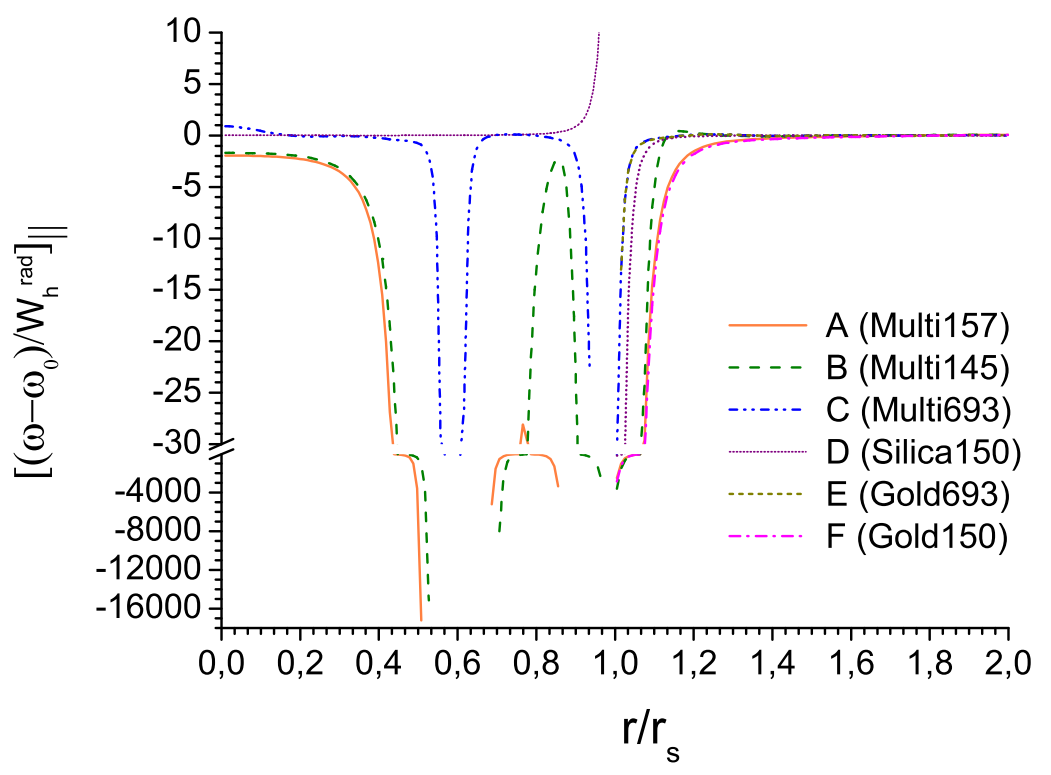

Figure 3: 


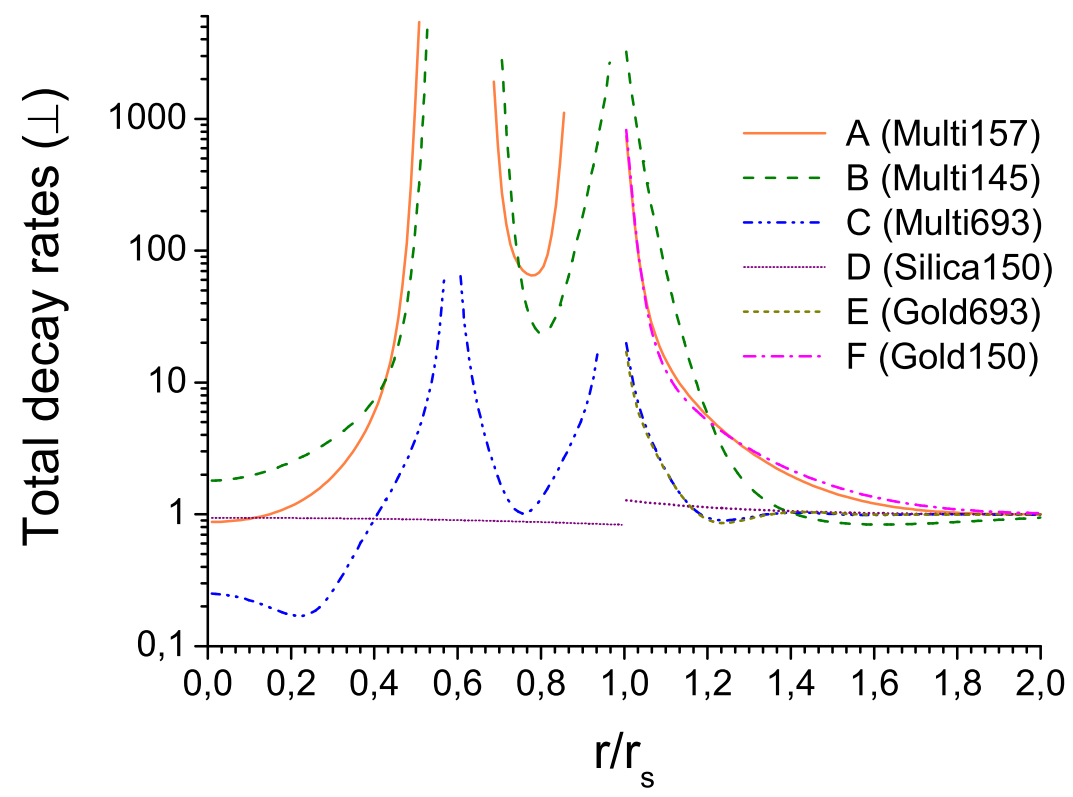

Figure 4:

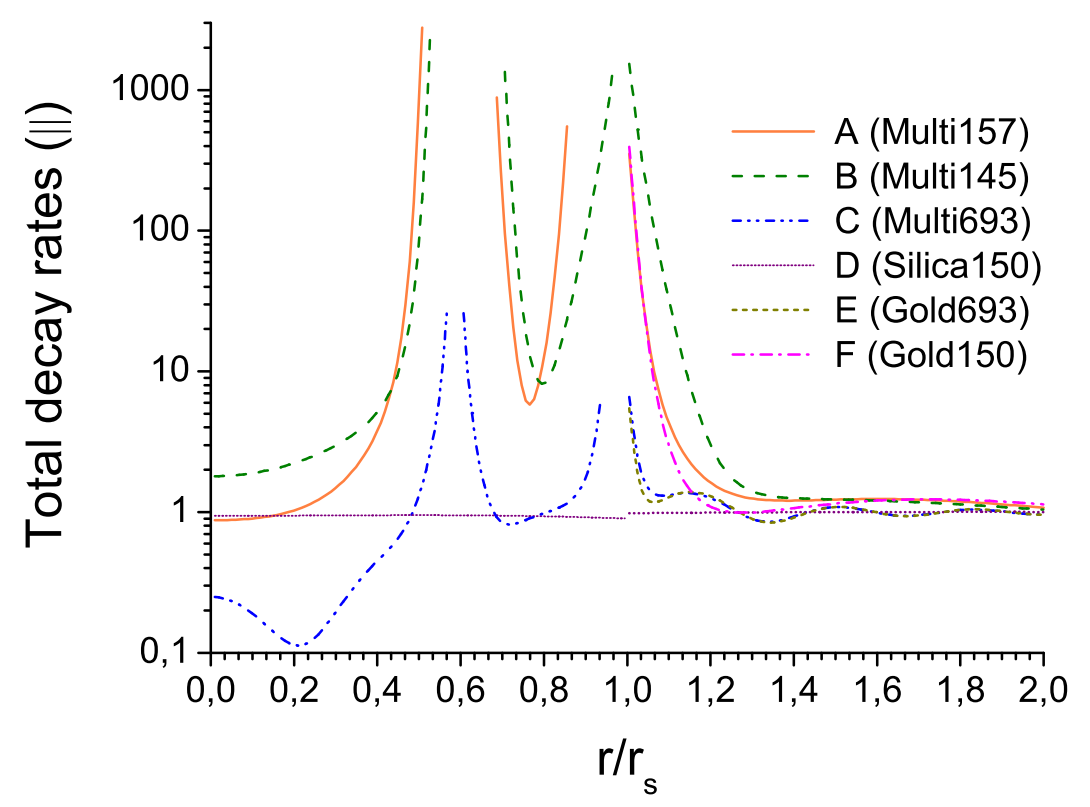

Figure 5: 


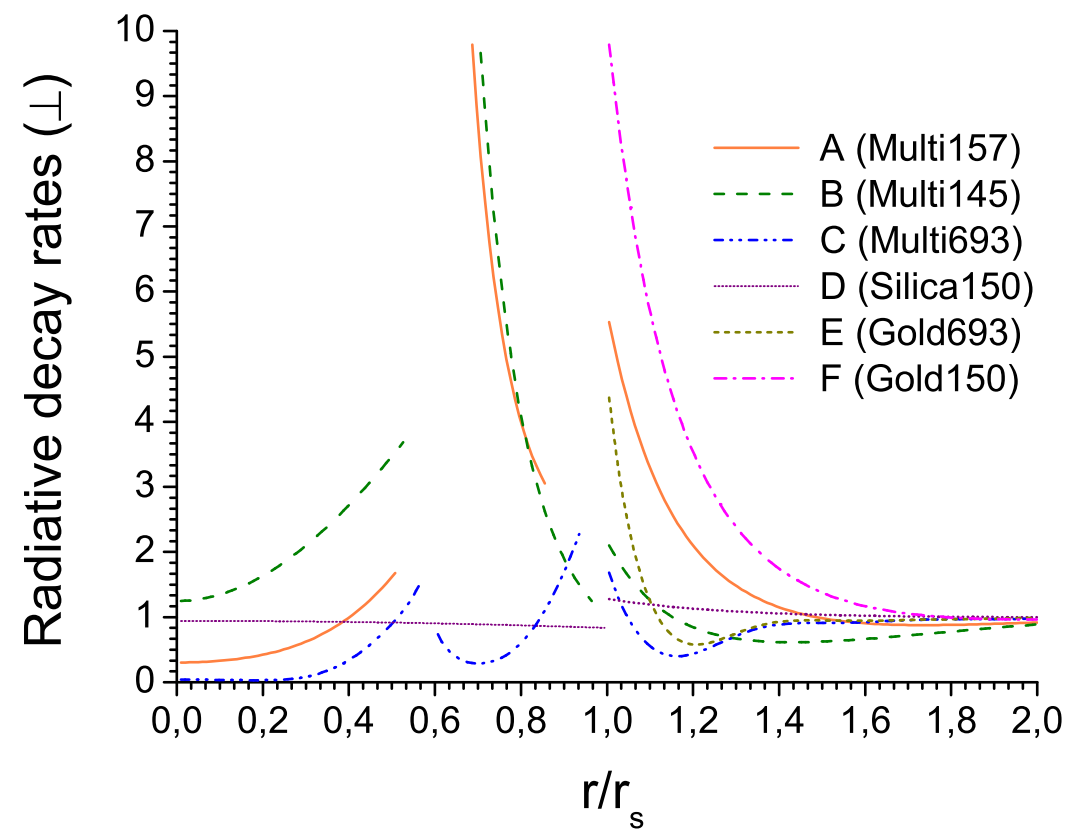

Figure 6: 


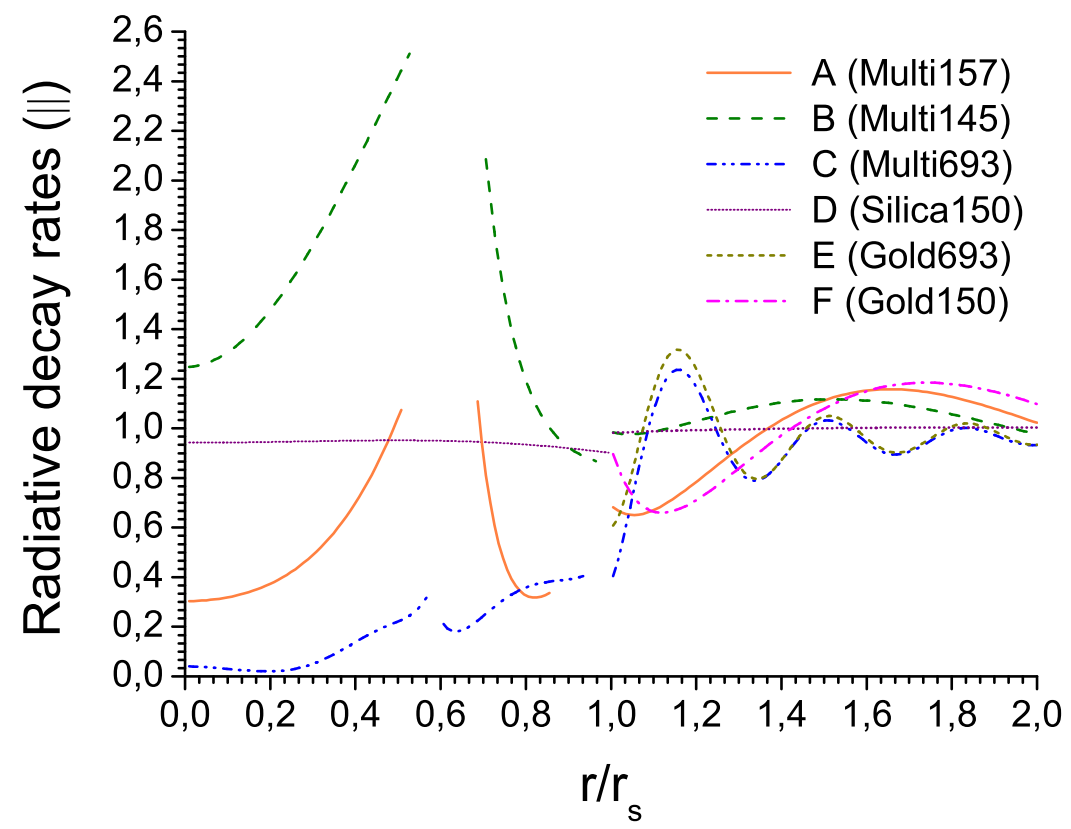

Figure 7: 


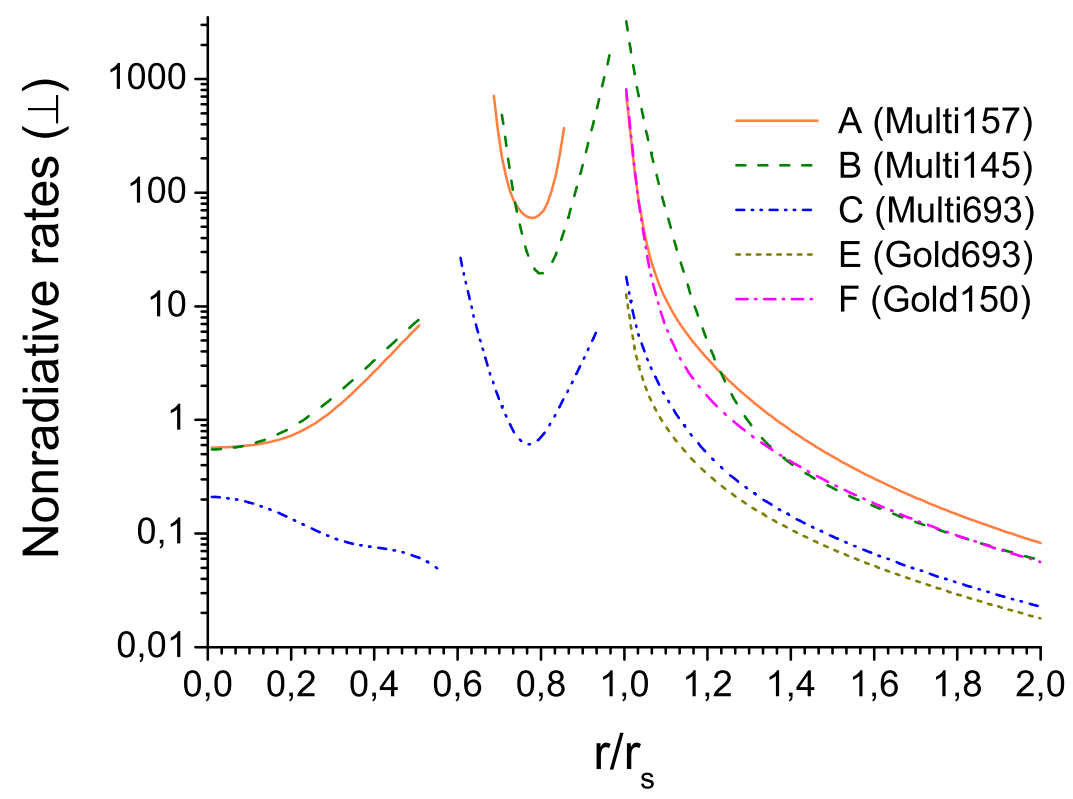

Figure 8:

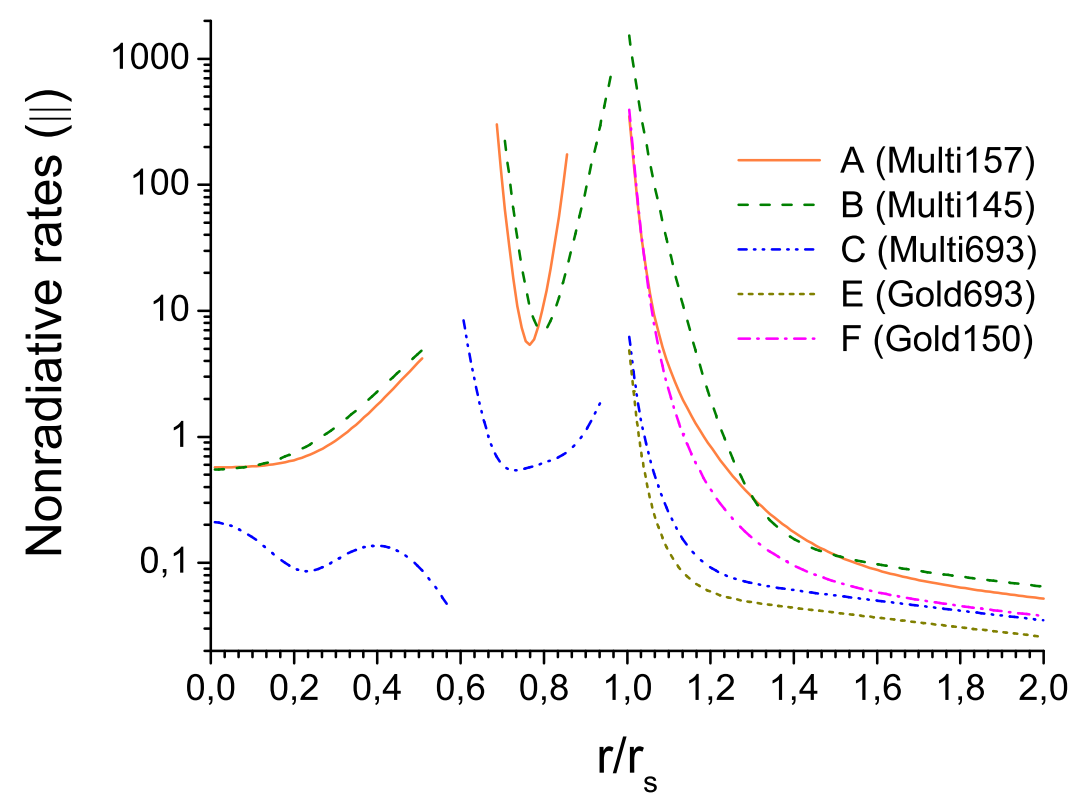

Figure 9: 


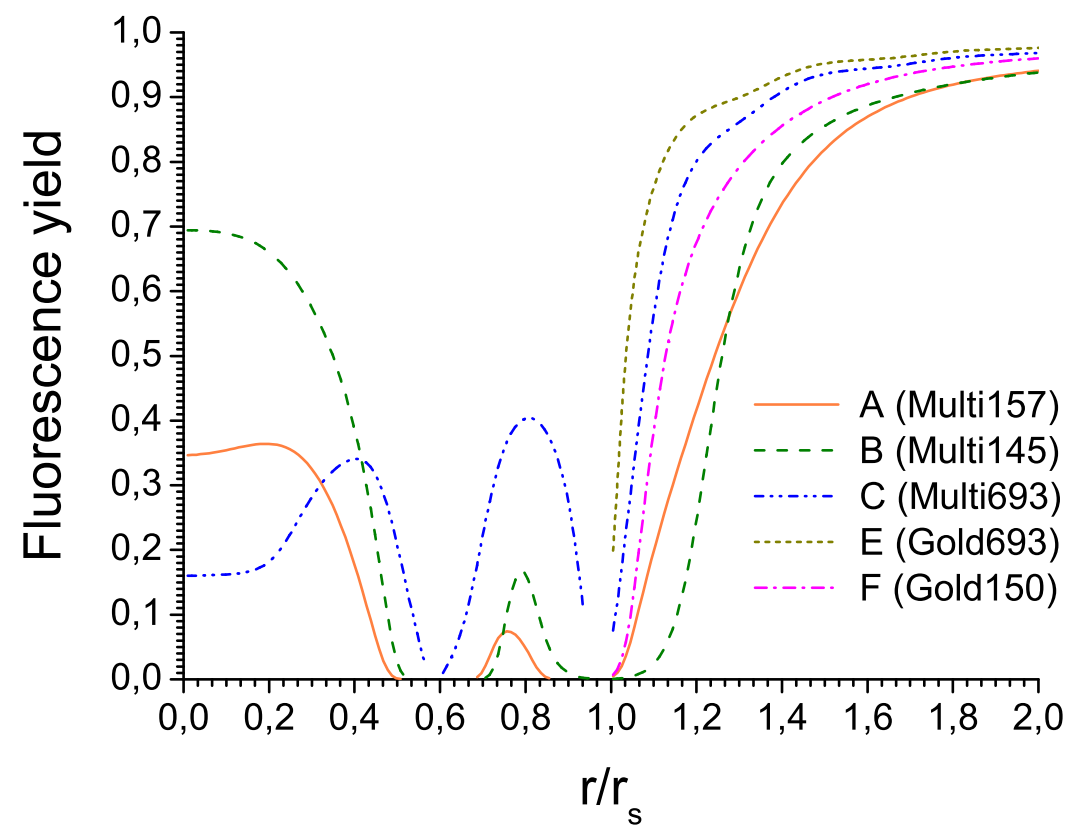

Figure 10: 


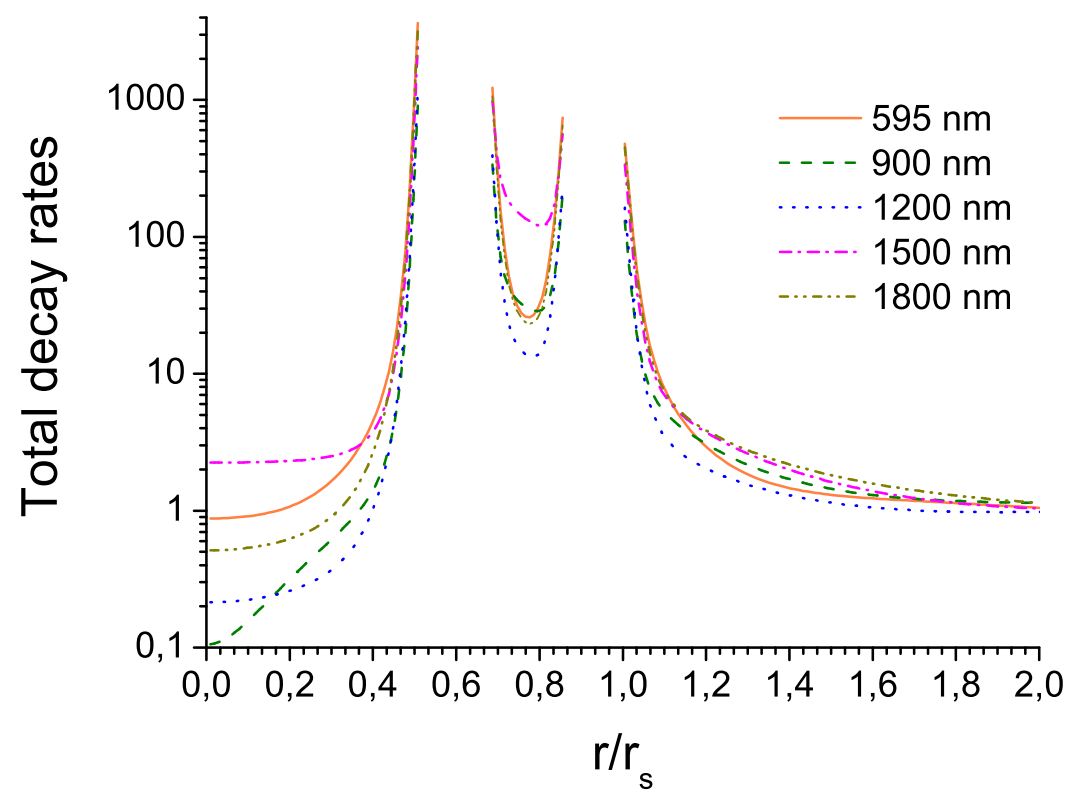

Figure 11: 